Exercices Exercices de rhétorique

de rhétorique $\quad 6 \mid 2016$

Sur l'épistolaire

\title{
Les grands romans de Mme de Sévigné
}

\section{Lise Charles}

\section{(2) OpenEdition}

Journals

Édition électronique

URL : http://journals.openedition.org/rhetorique/433

DOI : 10.4000/rhetorique.433

ISSN : 2270-6909

\section{Éditeur}

UGA Éditions/Université Grenoble Alpes

\section{Édition imprimée}

ISBN : 978-2-84310-316-2

\section{Référence électronique}

Lise Charles, "Les grands romans de Mme de Sévigné », Exercices de rhétorique [En ligne], 6 | 2016, mis en ligne le 10 février 2016, consulté le 12 septembre 2020. URL : http://journals.openedition.org/ rhetorique/433 ; DOI : https://doi.org/10.4000/rhetorique.433

Ce document a été généré automatiquement le 12 septembre 2020.

\section{(c) (i) (2)(2)}

Les contenus de la revue Exercices de rhétorique sont mis à disposition selon les termes de la Licence Creative Commons Attribution - Pas d'Utilisation Commerciale - Partage dans les Mêmes Conditions 4.0 International. 


\title{
Les grands romans de Mme de Sévigné
}

\author{
Lise Charles
}

\section{L'écriture et l'ennui}

1 Mme de Sévigné passe la majeure partie de l'année 1671 loin de Paris. Elle a beau demander à sa fille, dans sa lettre du 23 août : « ne craignez pas pour moi l'ennui que me peut donner la solitude » (I, 3281), elle paraît bien souvent désœuvrée. Elle a ainsi le temps de lire, et même de relire, des romans interminables, comme la Cléopâtre de La Calprenède (douze volumes, quinze mille pages). Elle a également le temps d'écrire des lettres, de longues lettres, des lettres parfois si longues qu'elles lui évoquent ces grands romans baroques que sa fille n'aime pas :

Voici une lettre d'une telle longueur que je vous pardonne de ne la point achever ;

je le comprendrai plus aisément que de demeurer au septième tome de Cassandre et

de Cléopâtre [...]. (28 juin 1671 : I, 284)

2 Pourquoi donc ne pas achever les lettres de sa mère serait-il plus pardonnable que de s'interrompre au milieu d'un roman de La Calprenède ? Sans doute parce que les lettres de Mme de Sévigné n'ont a priori en commun avec les romans baroques que leur longueur excessive, et ne contiennent rien de ce qui vous entraîne, pour reprendre l'expression de l'épistolière, "comme une petite fille ${ }^{2}$ »: nulle reconnaissance prodigieuse, nul berger trahi par sa bergère, et presque aucun coup d'épée. Mais Mme de Sévigné ne déteste rien tant que d'ennuyer sa fille (qui, à dire le vrai, paraît s'ennuyer facilement dans ses lectures) et elle semble recourir, pour susciter son intérêt, à des stratégies de suspens et de mise en intrigue qui sont, paradoxalement, en partie héritées du roman baroque. «Paradoxalement », car qui veut comparer l'écriture de Mme de Sévigné avec les stratégies du grand roman doit se confronter à trois problèmes. Tout d'abord, celui du matériau: Mme de Sévigné prétend toujours s'en tenir au vrai, et dispose donc d'un matériau relativement pauvre, au contraire de celui de la fiction, potentiellement illimité. Vient ensuite la question de la structuration du récit : Mme de Sévigné ne pouvant pas prévoir ce qui va se passer d'une lettre à l'autre, 
il ne saurait a priori pas y avoir d'organisation du récit à une échelle plus vaste que celle de la lettre. Le dernier problème est celui de l'énonciation: nous ne disposons que d'une moitié de l'échange épistolaire, donc d'un texte fondamentalement lacunaire, qui repose sur l'allusion, la connivence, et dont il serait raisonnable de penser qu'il ne peut pas se tenir en lui-même.

\section{Le vrai et le faux}

3 Le roman baroque relève non seulement du domaine de la fiction, mais de la fiction invraisemblable; c'est d'ailleurs l'un des reproches esthétiques qu'on lui adresse à partir de la seconde moitié du siècle ${ }^{3}$. Mme de Sévigné, elle, pratique une écriture du vrai: elle a un donné qu'elle ne peut pas en principe modifier, et qui est particulièrement pauvre durant cette année 1671. La mort de Vatel et le mariage manqué de Lauzun sont finalement peut-être les seuls événements de l'année qui aient une potentialité véritablement romanesque. Différentes stratégies permettent pourtant à l'épistolière de compenser la pauvreté de la réalité, autrement dit de compliquer et d'enrichir le donné.

4 Première voie d'entrée de la fiction : l'évocation de l'avenir. Parce qu'il n'existe pas encore, le futur constitue un point de contact possible entre la réalité et la fiction. Pour prendre un exemple fameux, Mme de Sévigné écrit dans sa lettre du 9 août 1671, au sujet de la grossesse de sa fille :

Votre petit garçon serait-il devenu fille ? Ne vous embarrassez pas; je vous aiderai à l'exposer sur le Rhône dans un petit panier de jonc, et puis elle abordera dans quelque royaume où sa beauté sera le sujet d'un roman. Me voilà comme Don Quichotte. Il y a d'horribles endroits dans Cléopâtre, mais il y en a de beaux [...]. (I, 317)

5 L'aventure imaginée fait référence au récit biblique de l'exposition de Moïse sur le Nil, mais renvoie également à l'univers des romans baroques, où il n'est pas rare de rencontrer des enfants voguant sur des barques : Cléandre est par exemple recueilli de cette manière dans Artamène ou Le Grand Cyrus de Madeleine de Scudéry ${ }^{4}$. C'est d'ailleurs Mme de Sévigné elle-même qui en parle comme du « sujet d'un roman » et qui enchaîne sans transition avec un commentaire sur la Cléopâtre; le glissement est peut-être également permis par le cadre égyptien commun à ce roman baroque et à l'histoire biblique. Imaginer par jeu le futur permet ainsi, en l'occurrence, de faire passer l'invraisemblable.

Mais la fiction peut aussi faire intrusion dans le récit d'événements passés. Ce sera alors sur le mode de l'irréel du passé, de ce qui aurait pu avoir lieu. Tout fait étant survenu à la place d'un autre qui aurait pu arriver, l'évocation du passé est encore une occasion pour Mme de Sévigné de multiplier les événements. Ainsi s'exclame-t-elle, dans sa lettre du 4 mars 1671 (I, 176) au sujet de la traversée du Rhône : « quel miracle que vous n'ayez pas été brisée et noyée dans un moment !»Ce faisant, elle dessine en négatif un scénario où Mme de Grignan aurait été brisée et noyée. Mme de Sévigné fait ici d'une pierre deux coups. Non seulement elle invente une histoire extraordinaire (la mort effroyable d'une jeune femme), mais elle présente ce qui s'est réellement passé comme plus extraordinaire que ce qui aurait pu se passer ; le vrai est en effet désigné comme un invraisemblable «miracle». Le cas n'est pas isolé; la plupart des événements racontés par Mme de Sévigné sont en fait des non-événements: Mme de Grignan ne s'est pas noyée, la fille de cette dernière n'est pas morte dans un incendie, le mariage de 
Lauzun avec la Grande Mademoiselle n'a pas eu lieu. Mais dire ce qui n'est pas, c'est donner à voir ce qui aurait pu être.

7 Enfin, et c'est a priori plus étonnant, la fiction contamine le récit de nouvelles actuelles. Dans sa lettre du 25 février 1671, Mme de Sévigné écrit : «M. Vallot est mort ce matin » (I, 170). Elle commence sa lettre du 27 février (I, 170) par : «Rien ne dure cette année, pas même la mort de M. Vallot. Il se porte bien, et au lieu d'être mort, comme on me l'avait dit, il a pris une pilule qui l'a ressuscité. » Les fausses morts sont un trait typique du roman baroque, emprunté directement au roman grec $^{5}$, si récurrent d'ailleurs que Charles Sorel s'en moque dans De la connaissance des bons livres: "L'on sera abusé ailleurs par de faux rapports, croyant qu'un homme ait esté tué ou noyé, pource qu'un autre de mesme nom l'a esté. ${ }^{6} »$ On pourrait multiplier les exemples : songeons ainsi, pour en citer un des plus fameux, à la fausse mort de Céladon au début de L'Astrée ${ }^{7}$. Certes, on pourra dire qu'à la différence de ce qui se passe dans le roman baroque, ce retournement n'a pas été calculé par l'épistolière. On répondra que Mme de Sévigné n'a pas pris soin de vérifier ses informations, peut-être comme pour laisser une place à la surprise. Comme l'écrit Laure Depretto, « en possession d'un scoop, l'épistolière baisse sa garde et ne garantit son information d'aucune source tangible ${ }^{8}$. On notera aussi que Mme de Sévigné, loin de s'excuser, comme on aurait pu s'y attendre, d'avoir transmis une fausse nouvelle, l'intègre en plaisantant à un double schéma, fictionnel, celui du mort ressuscité, et moral: son "rien ne dure » a en effet un accent de prédicateur ou de moraliste.

8 Mais pour rendre intéressant le matériau, il n'est pas toujours besoin de faire entrer la fiction dans la narration; il suffit que le vrai soit présenté comme le serait une fiction. On en prendra ici un seul exemple, celui de l'incendie raconté par Mme de Sévigné dans sa lettre du 20 février $1671^{9}$. Commençons par lire l'incipit de la Cléopâtre :

Les ombres de la nuict n'avoient pas encore fait place à la lumiere, quand le triste Tyridate éveillé par ses cruelles inquietudes, \& ne pouvant attendre la clarté du jour, sortit de sa solitaire demeure, pour promener son corps languissant, \& ses amoureuses pensées sur le rivage d'Alexandrie [...]. Il avoit donné quelques momens à ces tristes considerations, lors que relevant ses yeux de sur la terre, où il les avoit tenus long temps attachez, \& les jettant sur la mer, ils furent d'abord frappez d'un object capable de les arrester. C'estoit un grand feu ${ }^{10}[. .$.$] .$

Voici maintenant le début de la lettre du 20 février 1671:

Je vous avoue que j'ai une extraordinaire envie de savoir de vos nouvelles. [...] Je me dévore, en un mot; j'ai une impatience qui trouble mon repos. Je suis bien assurée qu'il me viendra des lettres [...], mais je les attends, et je ne les ai pas. Il faut se consoler, et s'amuser en vous écrivant. Vous saurez, ma petite, qu'avant-hier, mercredi, après être revenue de chez $\mathrm{M}$. de Coulanges [...], je revins me coucher ; cela n'est pas extraordinaire. Mais ce qui l'est beaucoup, c'est qu'à trois heures après minuit, j'entendis crier au voleur, au feu [...]. Je vis la maison de Guitaut tout en feu [...]. (I, 163-164)

10 Ces incipit (de roman, de lettre) sont construits d'une manière semblable. Dans les deux cas s'enchaînent deux séquences: nous voyons d'abord une personne troublée par l'absence de l'objet aimé; ensuite est raconté un incendie qui a eu lieu en pleine nuit. Mais tandis que chez La Calprenède, l'enchaînement est chronologique, c'est un ordre artificiel chez Mme de Sévigné, qui semble rapprocher arbitrairement les deux séquences, avec une transition en apparence très faible et désinvolte: "Il faut se consoler, et s'amuser en vous écrivant ». L'épistolière compense pourtant l'absence de lien chronologique par des liaisons rhétoriques virtuoses. Ainsi, le thème du repos 
troublé lie, comme dans le roman, les deux séquences : le repos de Mme de Sévigné est d'abord troublé, de manière récurrente, par ses inquiétudes maternelles; le motif est ensuite réactualisé, puisque son repos est troublé accidentellement par l'incendie de la maison de Guitaut, qui survient pendant la nuit. Le lien est également fait par l'usage du verbe dévorer ( "Je me dévore »), qui annonce discrètement le motif de l'incendie, le verbe étant souvent employé pour désigner l'action du feu ${ }^{11}$. Le modèle de la Cléopâtre semble bien peser ici jusque dans l'enchaînement thématique artificiel de séquences qui étaient indépendantes dans la réalité; il donne une couleur romanesque non seulement à l'épisode de l'incendie, mais aussi, rétrospectivement, à la première séquence, l'ouverture de la lettre, qui ne contient pourtant aucune aventure.

Il arrive même qu'une teinte romanesque soit projetée sur l'ordinaire sans que la lettre contienne aucun événement extraordinaire. C'est le dernier artifice de Mme de Sévigné : faire croire qu'elle possède un matériau alors même qu'elle n'en a pas, autrement dit créer de toutes pièces l'événement. Encore une fois, on se contentera d'en prendre un exemple, celui du début de la lettre du 16 septembre 1671 :

Je suis méchante aujourd'hui, ma bonne; je suis comme quand vous me disiez:

"Vous êtes méchante. » Je suis triste; je n'ai point de vos nouvelles. La grande amitié

n'est jamais tranquille, MAXIME. (I, 345)

Cinq segments s'enchaînent ici en parataxe :

1. Je suis méchante, aujourd'hui, ma bonne; //2. Je suis comme quand vous me disiez: "Vous êtes méchante». //3. Je suis triste;//4.Je n'ai point de vos nouvelles. // 5. La grande amitié n'est jamais tranquille, MAXIME.

13 Le premier segment appelle une explication : «je suis méchante » a un sens très vague, on ne sait pas si Mme de Sévigné veut dire qu'elle est de méchante humeur, ou qu'elle s'est conduite de manière méchante, et on n'en connaît pas la raison. Le deuxième segment est à peu près redondant, donc déceptif. Puis le troisième, toujours en parataxe: «je suis triste». On a enfin une réponse: Mme de Sévigné est méchante parce qu'elle est triste. Mais le problème est encore retardé, selon une logique récursive : pourquoi est-elle triste ? se demande-t-on alors. Ce n'est qu'à la fin que nous pouvons reconstituer le raisonnement dans son entier : je suis méchante parce que je suis triste, je suis triste parce que je n'ai point de vos nouvelles. Rien de neuf, en somme. Le raisonnement est clos par une maxime, comme on en trouve souvent pour ouvrir ou fermer une séquence dans les grands romans. Mme de Sévigné met donc en intrigue ses sentiments; elle fait un roman sur le rien, ou du moins sur l'ordinaire.

\section{Questions de structure}

Les romans baroques paraissent toujours en plusieurs livraisons, souvent sur de nombreuses années. Ainsi la première publication de la Cléopâtre s'est-elle faite sur dix ans, de 1648 à 1658. Marc Escola parle de "fictions périodiques" pour désigner ces fictions qui commencent à paraître "sans que l'auteur [...] dispose d'un manuscrit achevé ni d'une idée bien nette des "parties" suivantes et du dénouement de l'intrigue ${ }^{12}$ ». Ces œuvres sont donc écrites «dans l'ignorance de la fin ${ }^{13}$ ». Bien entendu, il s'agit également d'une caractéristique de l'écriture épistolaire en général; mais le rapprochement, en apparence, s'arrête là : chaque partie d'un roman baroque est évidemment beaucoup plus vaste qu'une lettre, si longue soit-elle. En outre, si les fictions périodiques sont souvent écrites dans l'ignorance de leur fin, cette ignorance n'est ni inhérente au genre, ni absolue : l'auteur d'un roman baroque sait au moins 
d'une manière vague dans quelle direction il se dirige, ne serait-ce qu'en raison des fortes contraintes génériques qui guident la composition. Dans l'écriture épistolaire non fictionnelle, au contraire, la construction du récit ne peut a priori se faire qu'à une échelle beaucoup plus restreinte, celle de la lettre, puisque l'auteur ne peut pas prévoir ce qui va se passer par la suite.

Il n'est donc pas déraisonnable de comparer la structure de certaines lettres de Mme de Sévigné à une structure romanesque, mais cela implique d'y voir une opération d'extrême miniaturisation. Plusieurs lettres ont ainsi un incipit typique de celui des grands romans, à savoir l'ouverture in medias res, suivie d'une analepse. Ainsi de la lettre du 19 décembre 1670 :

Ce qui s'appelle tomber du haut des nues, c'est ce qui arriva hier soir aux Tuileries ;

mais il faut reprendre les choses de plus loin. (I, 140 ${ }^{14}$ )

De même, l'inclusion dans les lettres de nombreuses histoires et leur délimitation par des marquages textuels forts ${ }^{15}$ peuvent faire penser à la technique de l'enchâssement, typique des romans baroques. Enfin, les énigmes et autres procédés de suspension, qui contribuent dans les romans baroques à fixer les bornes de séquences souvent assez vastes, fonctionnent comme en accéléré chez Mme de Sévigné. Dans la lettre du 13 mars 1671, par exemple, elle écrit :

$\mathrm{Au}$ reste, si vous croyez les filles de la Reine enragées, vous croirez bien. Il y a huit jours que Mme de Ludres, Coëtlogon et la petite de Rouvroy furent mordues d'une petite chienne, qui était à Théobon. Cette petite chienne est morte enragée [...]. (I, 184)

17 La première phrase est une énigme, elle ressemble beaucoup aux paroles oraculaires si fréquentes dans les romans baroques. Sa résolution repose, comme celle de nombreux oracles, sur une syllepse de sens: on pensait d'abord qu'il fallait prendre l'adjectif enragées au sens figuré, on s'aperçoit qu'il faut le prendre au sens propre. Mais tandis que les oracles des romans baroques sont d'ordinaire expliqués au moins une centaine de pages après avoir été énoncés, délimitant ainsi de très longues séquences, le mystère est ici résolu presque immédiatement.

Il n'est pourtant pas vrai de dire que la structuration ne saurait se faire au-delà de la lettre, que Mme de Sévigné ne peut rien prévoir de ce qu'elle aura à écrire dans ses lettres suivantes. Certes, l'effet de continuité est sans doute renforcé pour le lecteur moderne, qui, tout simplement grâce à l'édition, est en mesure de lire les lettres l'une après l'autre. Mais ce n'est pas le seul facteur de continuité. Là encore, il faut distinguer différentes catégories.

19 Un premier type d'éléments relève, on l'a vu, de l'imprévu. Ces éléments, comme le récit de l'incendie ou celui des filles de la reine enragées, sont extrêmement nombreux et divers, et ce sont eux qui donnent aux lettres leur côté hétéroclite. Mais il existe aussi un second type d'éléments, dont l'imprévu est, pour ainsi dire, prévu. On sait qu'il va se passer quelque chose sur tel sujet, mais on ne sait pas quoi. Ainsi, on sait que Mme de Grignan doit accoucher, mais on ne sait ni si l'accouchement sera heureux, ni s'il en naîtra un garçon ou une fille; on sait que l'extraordinaire projet de mariage de Mademoiselle avec Lauzun aura une suite, mais on ignore laquelle. Ce genre d'événements permet à Mme de Sévigné de clore ses lettres sur des annonces comme : "Adieu; les lettres qui sont portées par cet ordinaire vous feront voir si nous disons vrai ou faux" (lettre à Coulanges du 15 décembre 1670: I, 140). Ces promesses qui ferment la lettre tout en l'ouvrant sur la suivante ne sont pas sans rappeler celles qui 
terminent fréquemment les chapitres de roman. D’autres éléments sont, eux, tout à fait prévus. Ainsi, Mme de Sévigné sait pertinemment qu'elle va rester plusieurs mois éloignée de sa fille, et le discours de la douleur peut servir de basse continue à l'ensemble des lettres de l'année 1671. Il est frappant que cette basse continue, qui fait véritablement le lien entre l'ensemble des lettres, constitue également presque toujours la trame principale des romans grecs, donc des romans baroques: deux personnages s'aiment tendrement, sont séparés, et ont l'espoir de se retrouver.

Ugo Dionne, dans La Voie aux chapitres, considère que l'entrelacement et le récit inséré sont les deux principaux facteurs de "prolifération narrative » du roman d'Ancien Régime $^{16}$. Il semble que ce soient également ceux des lettres de Mme de Sévigné. Une ligne directrice sert de trame (les déclarations de tendresse de Mme de Sévigné à sa fille); cette ligne est interrompue régulièrement, sur l'ensemble de la correspondance, par des lignes secondaires (par exemple les histoires d'Adhémar), qui constituent autant d'intrigues parallèles et entrelacées; elle est interrompue plus ponctuellement par des histoires enchâssées brèves et généralement encadrées par des marquages textuels forts.

\section{Sévigné, sa fille et nous}

Les lettres de Mme de Sévigné pourraient-elles alors être lues comme un roman? Après tout, si elles n'ont ni début ni fin, elles ne diffèrent pas en cela du roman baroque, qui commence la plupart du temps par le milieu, et qui ne se finit pas toujours: L'Astrée d'Honoré d'Urfé est inachevée, ainsi que le Faramond de La Calprenède et beaucoup d'autres. Mais il existe une objection plus forte à cette tentation de lecture décalée : le texte que nous lisons est fondamentalement lacunaire et allusif, puisqu'il manque une moitié de l'échange épistolaire. Et son caractère énigmatique, son mystère, n'est pas dû à un calcul pour ménager l'intérêt du lecteur, mais, beaucoup plus trivialement, à un accident, la perte des lettres de Mme de Grignan.

Or, une lecture effective de ces lettres nous donne pourtant l'étonnante impression qu'elles ont une certaine autonomie. Nous ne nous demanderons pas si l'épistolière écrivait de fait pour ce que Bernard Bray appelle un "public secondaire ", c'est-à-dire « un cercle de lecteurs débordant le groupe des amis intimes du destinataire ${ }^{17}$ ». Il nous suffira de rappeler que, même si l'on n'a pas l'intention d'être lu par d'autres, on peut écrire en s'imaginant que d'autres nous lisent. C'est ce qui semble se passer quand Mme de Sévigné romance des événements que sa fille connaît déjà, pour les avoir vécus. La lettre du 4 mars 1671 sur la traversée du Rhône en est un bon exemple; Mme de Sévigné y raconte, au présent de narration, ce que sa fille lui a déjà raconté :

Et M. de Grignan vous laisse conduire la barque ! et quand vous êtes téméraire, il trouve plaisant de l'être encore plus que vous! [...] Un tourbillon de vent vous jette violemment sous une arche! (I, 175-176)

Certes, la répétition partielle des lettres reçues est un passage presque obligé de l'échange épistolaire; c'est une manière de dire qu'on s'intéresse à l'autre, et de commenter point par point ce qui nous a été raconté. Mais ici, le procédé excède la simple politesse, il est poussé à l'extrême, de sorte que les lettres de la mère permettent toujours au tiers lecteur de lire, en filigrane, les lettres de la fille. 

extraordinaires que la quasi-noyade de Mme de Grignan. Voici comment Mme de Sévigné commence sa lettre du 9 février 1671 :

Je reçois vos lettres, ma bonne, comme vous avez reçu ma bague. Je fonds en larmes en les lisant ; il semble que mon cœur veuille se fendre par la moitié. Il semble que vous m'écriviez des injures ou que vous soyez malade ou qu'il vous soit arrivé quelque accident, et c'est tout le contraire. Vous m'aimez, ma chère enfant, et vous me le dites d'une manière que je ne puis soutenir sans des pleurs en abondance [...]. (I, 151-152)

La première phrase, «je reçois vos lettres [...] comme vous avez reçu ma bague », est énigmatique pour un tiers lecteur, car elle repose sur une allusion: ne sachant pas comment la fille a reçu la bague de sa mère, nous ignorons comment la mère reçoit les lettres de sa fille. Cependant, nul n'est besoin pour Mme de Sévigné d'expliquer la chose à Mme de Grignan, puisque cette dernière sait bien évidemment comment elle a reçu la bague en question. L'explication est pourtant donnée à la phrase suivante : « Je fonds en larmes en les lisant; il semble que mon cœur veuille se fendre par la moitié. » Nous savons maintenant que Mme de Sévigné a pleuré en recevant les lettres, mais le problème n'est que reporté, puisque nous ignorons pourquoi. L'épistolière prend alors soin de se mettre explicitement à notre place, c'est-à-dire à la place de quelqu'un qui lirait sa lettre et qui ne serait pas Mme de Grignan : «Il semble que vous m'écriviez des injures ou que vous soyez malade ou qu'il vous soit arrivé quelque accident, et c'est tout le contraire. » Le glissement d'un point de vue à l'autre est permis par la polysémie du verbe sembler : «il semble que mon cœur [...] » signifiait presque «j'ai l'impression que mon cœur [...] », tandis que « il semble que vous m'écriviez des injures » équivaut à « on dirait en me lisant que vous m'écriviez des injures ». La résolution complète du mystère, qui n'en était un que pour nous (Mme de Grignan sait qu'elle n'a pas écrit d'injures à sa mère), est donnée à la phrase suivante : « Vous m'aimez, ma chère enfant [...] ». On a vu plus haut d'autres exemples de constructions à retardement, fondées sur la récursivité : elles sont très fréquentes chez Mme de Sévigné ; mais ce qu'il y a d'étonnant ici, c'est que l'effet de suspension ne fonctionne que pour le tiers lecteur que nous sommes. Comme si Mme de Sévigné s'inventait un public secondaire.

Bref, le suspens paraît susceptible de prendre trois formes. Il peut porter sur des événements futurs (le mariage de Lauzun aura-t-il lieu? Mme de Grignan accouchera-telle d'un fils ?) : Mme de Sévigné, Mme de Grignan et le tiers lecteur sont alors dans l'incertitude. Il peut également porter sur des événements passés, connus de la seule Mme de Sévigné (pourquoi les filles de la reine sont-elles enragées ?) : Mme de Grignan et le tiers lecteur sont alors dans l'incertitude. Il peut enfin porter sur des événements connus de Mme de Sévigné et de sa fille: nous sommes alors les seuls à être dans l'incertitude ${ }^{18}$. Plus généralement, on peut constater la grande lisibilité des lettres de Mme de Sévigné, qui paraît toujours prendre soin, consciemment ou non, d'un tiers lecteur. Tout se passe presque comme si l'épistolière, ayant prévu que les lettres de Mme de Grignan allaient se perdre, faisait attention à ce que ses lettres contiennent tout en elles-mêmes, et puissent se lire non comme un roman auquel on aurait arraché une page sur deux, mais bien comme un roman complet. 


\section{D'autres écueils}

à qui j'écris ont intérêt que je lise de bons livres ", déclare Mme de Sévigné dans sa lettre du 7 octobre 1671 (I, 360). Le roman baroque, qui constitue une des lectures favorites de l'épistolière durant cette année, semble en effet fonctionner également pour elle comme un modèle littéraire, auquel elle emprunte certaines stratégies afin de déjouer le risque d'ennui suscité par la longueur et la fréquence de ses lettres. Mais ne risque-t-elle pas, en voulant éviter un écueil, de tomber dans un autre? Les grands romans, à l'époque où écrit Mme de Sévigné, sont loin d'être considérés comme de «bons livres"; ils sont presque devenus une littérature honteuse, dont la lecture suscite, pour reprendre l'expression de Delphine Denis, une certaine «mauvaise conscience ${ }^{19}$ ». L'opposition est double : sur le plan esthétique, on reproche notamment au grand roman l'embarras de sa construction et ses invraisemblances; sur le plan moral, un auteur comme Pierre Nicole, dont Mme de Sévigné est d'ailleurs une lectrice assidue, ne cesse de critiquer la mauvaise influence des romans sur les mœurs ${ }^{20}$. Mme de Sévigné est une femme de son temps, elle connaît tous ces reproches, et elle a soin de prendre ses distances par rapport au modèle même qu'elle imite. Elle le fait d'abord par une critique théorique à l'égard des grands romans, qui sont, dit-elle, écrits dans un style effroyable, et qu'elle ne lit que par désœuvrement. Mais le meilleur moyen de se prémunir contre l'accusation de mauvais goût, c'est bien sûr de briser ses propres élans baroques par l'humour ou la désinvolture, qui marquent souvent l'ouverture et la clôture de ses récits. Ainsi, le récit de l'incendie est introduit, on l'a vu, par un très désinvolte "il faut [...] s'amuser en vous écrivant", et se clôt par une réécriture burlesque du même événement :

Mais si on avait pu rire dans une si triste occasion, quels portraits n'aurait-on point fait de l'état où nous étions tous? Guitaut était nu en chemise, avec des chausses.

Mme de Guitaut était nu-jambes, et avait perdu une de ses mules de chambre.

L'imitation, alors, se change en une parodie comme on pourrait en lire chez Scarron ou Furetière; pourtant, contrairement à un Scarron, Mme de Sévigné se moque alors moins du roman baroque que de son propre côté baroque.

Et c'est une faiblesse qui revient toujours au galop: les imitations peuvent en effet survenir aux endroits où on les attend le moins. Ainsi, dans sa lettre du 12 juillet 1671, après avoir détaillé son attirance mêlée de dégoût pour les romans de la Calprenède, auxquels elle se laisse prendre "comme à de la glu», elle voit soudain sa fille surgir devant elle : «Vous m'apparaissez pour me faire honte; mais je me dis de méchantes raisons, et je continue " $(\mathrm{I}, 294)$. Or, au moment même où l'épistolière dit sa honte par rapport à ses lectures, elle les prend encore pour modèles. Cette "apparition » fantomatique et menaçante d'une personne absente est en effet un motif typique des romans baroques : dans la dernière partie de la Cléopâtre, par exemple, César apparaît en songe à Auguste pour lui faire des reproches ${ }^{21}$.

Il faudra encore dix-huit ans à Mme de Sévigné pour parvenir à une déclaration plus apaisée au sujet des romans. C'est en 1689 qu'elle écrit à sa fille :

Il y a des exemples des bons et des mauvais effets de ces sortes de lectures. Vous ne les aimez pas ; vous avez fort bien réussi. Je les aimais ; je n'ai pas trop mal couru ma carrière. (16 novembre 1689 : III, 757) 
31 Des goûts et des couleurs, en somme. Notons cependant qu'ent
fait carrière en littérature, c'est bien celle qui aimait les romans.

\section{NOTES}

1. Données entre parenthèses, les références aux lettres de Mme de Sévigné mentionnent la date de la lettre et sa pagination (tome et page) dans l'édition de référence: Correspondance, éd. R. Duchêne, Paris, Gallimard, coll. « Bibliothèque de la Pléiade », 1972-1978, 3 tomes.

2. «La beauté des sentiments, la violence des passions, la grandeur des événements, et le succès miraculeux de leurs redoutables épées, tout cela m'entraîne comme une petite fille [...]» (12 juillet $1671: \mathrm{I}, 294$ ).

3. Du Plaisir, théoricien de la nouvelle galante, écrira ainsi que ce qui a «fait haïr " les anciens romans, c'est « leur longueur prodigieuse, le mélange de tant d'histoires diverses, leur grand nombre d'acteurs, la trop grande antiquité de leurs sujets, l'embarras de leur construction, leur peu de vraisemblance, l'excès dans leur caractère » (Sentiments sur les lettres et sur l'histoire avec des scrupules sur le style, dans Poétiques du roman: Scudéry, Huet, Du Plaisir et autres textes théoriques et critiques du XVII ${ }^{e}$ siècle sur le genre romanesque, éd. Camille Esmein, Paris, Champion, 2004, p. 762).

4. Paris, Augustin Courbé, 1656, partie 4, livre 1, p. 39.

5. Voir par exemple la fausse mort de Callirhoé, frappée par Chairéas, dans le roman de Chariton (Chairéas et Callirhoé, éd. G. Molinié, Paris, Les Belles Lettres, 1979).

6. De la connaissance des bons livres ou examen de plusieurs autheurs, présentation d'Hervé D. Béchade, Genève-Paris, Slatkine, 1981, p. 118.

7. Pour d'autres exemples, voir G. Molinié, Du roman grec au roman baroque, Toulouse, Presses universitaires du Mirail, 1995, p. 261-278.

8. «Annoncer l'incertain : les fausses nouvelles dans les Lettres de Mme de Sévigné », Littératures classiques, $\mathrm{n}^{\circ}$ 71, 2010, p. 229.

9. L'épisode de l'incendie est rapproché par Chrystelle Barbillon de la scène d'ouverture du Grand Cyrus (« "Me voilà comme Don Quichotte”, jeux et enjeux des modèles fictionnels romanesques et théâtraux ", dans Lectures de Mme de Sévigné, op. cit., p. 141). Il est très courant en effet qu'un événement spectaculaire, naufrage, carnage ou incendie, constitue l'incipit des romans baroques (encore un héritage du roman grec).

10. La Calprenède, Cléopâtre, Paris, chez la veuve Nicolas de Cergy, 1652, livre I, p. 1-2.

11. À l'entrée dévorer de la première version du Dictionnaire de l'Académie française (1694), on lit ainsi : «On dit fig. Le feu devore, le temps devore tout. »

12. «Le clou de Tchekhov, retours sur le principe de causalité régressive », dans La Partie et le tout. La Composition du roman de l'âge baroque au tournant des Lumières, études réunies et présentées par M. Escola, J. Herman, L. Omacini, P. Pelckmans et J.-P. Sermain, Louvain, Peeters, 2011, p. 107-117, ici p. 117.

13. Voir par exemple l'article «Longueur de Cleveland", dans J.-P. Sermain éd., Cleveland de Prévost, l'épopée du XVIII siècle, Paris, Desjonquères, 2006, p. 181-203.

14. L'exemple est cité par Christine Noille, « Les lettres de Sévigné sont-elles informes ? Éléments pour une rhétorique de la disposition ", dans Lectures de Madame de Sévigné, les lettres de 1671, op. cit., p. 35. 
15. De nombreuses séquences, par exemple, sont ouvertes par le présentatif voici, suivi d'une relative périphrastique, et closes par un voilà. Voir à ce sujet l'article de Stéphane Macé, " "Voilà bien des lanternes, ma pauvre bonne", quelques remarques sur l'emploi des présentatifs voicivoilà ", dans Lectures de Madame de Sévigné, op. cit., p. 177-187.

16. La Voie aux chapitres. Poétique de la disposition romanesque, Paris, Seuil, coll. « Poétique », 2008, p. 451.

17. "L'épistolier et son public en France au XVII ${ }^{\mathrm{e}}$ siècle ", Travaux de linguistique et de littérature, 1973, p. 11. Voir aussi Fritz Nies, Les Lettres de Madame de Sévigné. Conventions du genre et sociologie des publics, trad. Michèle Creff, Paris, Champion, coll. « Lumière classique ", 2001.

18. Les théoriciens modernes parleraient dans ces deux derniers cas de curiosité. Ainsi, selon Tzvetan Todorov («Typologie du roman policier», dans Poétique de la prose, Paris, Seuil, 1971, p. 60), il y a curiosité quand on se demande, dans un mouvement rétrospectif : que s'est-il passé ? et il y a suspense quand on se demande, dans un mouvement prospectif : que va-t-il se passer? Voir aussi Raphaël Baroni, La Tension narrative. Suspense, curiosité et surprise, Paris, Seuil, coll. «Poétique ", 2007, notamment p. 100-110.

19. «[...] à la différence de l'adhésion obtenue comme de force par la lecture, le jugement émis après coup ménage une part d'auto-ironie, moment de retour critique sur l'enthousiasme ressenti. Dans cette distance subtile de soi à soi, qui autorise un abandon consenti en connaissance de cause aux séductions du romanesque, s'épanouit en quelque sorte, entre indulgence amusée et mauvaise conscience, le plaisir que repérait en elle Mme de Sévigné à la lecture des "sottises" romanesques de La Calprenède." («Romanesque et galanterie», dans Le Romanesque, éd. G. Declercq et M. Murat, Paris, Presses Sorbonne nouvelle, 2004, p. 114.)

20. On y lit par exemple : « Non seulement les comedies \& les romans rendent l'esprit mal disposé pour toutes les actions de religion \& de piété, mais il en conçoit du dégoût pour toutes les actions sérieuses \& communes. [...] on y prend insensiblement une disposition d'esprit toute romanesque, on se remplit la tête de heros \& de heroïnes : \& les femmes principalement [...] s'impriment tellement dans la fantaisie cette sorte de vie, que les petites affaires de ménage leur deviennent insupportables" (traité «De la comédie», dans Essais de morale, contenus en divers traités sur plusieurs devoirs importans, vol. I, Paris, Guillaume Desprez, 1733, p. 265-266).

21. La Calprenède, Cléopâtre, op. cit., partie XII, livre 4, p. 467.

\section{AUTEUR}

\section{LISE CHARLES}

Université Paris-Sorbonne / STIH-EA 4509 